03

\title{
Влияние температуры носовой части конуса на спектры возмущений в гиперзвуковом пограничном слое
}

\author{
(С) Д.А. Бунтин ${ }^{1,2}$, А.А. Маслов ${ }^{1,2}$, Ю.В. Громыко ${ }^{1,2}$ \\ ${ }^{1}$ Институт теоретической и прикладной механики им. С.А. Христиановича \\ СО РАН, Новосибирск \\ ${ }^{2}$ Новосибирский государственный университет \\ E-mail: bountin@itam.nsc.ru
}

Поступило в Редакцию 28 апреля 2017 г.

\begin{abstract}
Представлены результаты экспериментального исследования влияния локального нагрева/охлаждения затупленной носовой части конуса на развитие возмущений в пограничном слое при числе Маха $M=6$. Выполнен сравнительный анализ спектров пульсаций давления на поверхности конуса. Показано, что в пограничном слое острого конуса нагрев/охлаждение приводит к дестабилизации/стабилизации возмущений второй моды. Для затупленных конусов наблюдается противоположный эффект: нагрев приводит к слабой стабилизации возмущений, охлаждение - к дестабилизации. Этот эффект усиливается при увеличении радиуса затупления носовой части конуса.
\end{abstract}

DOI: 10.21883/PJTF.2017.20.45145.16841

Разработка многоразовых и безопасных гиперзвуковых летательных аппаратов (ГЛА) требует полного контроля над распределением тепла по всей системе тепловой защиты. Если в каком-то месте на поверхности транспортного средства пограничный слой переходит из ламинарного состояния в турбулентное, то резко увеличиваются тепловые потоки к поверхности. В связи с этим проблема предсказания ламинарно-турбулентного перехода (ЛТП) является одной из ключевых проблем аэротермодинамики [1]. При полете высокоскоростных летательных аппаратов наиболее интенсивному нагреву подвергаются его носовые части. Возникающие при этом зоны тепловых неоднородностей могут оказать существенное влияние на пограничный слой, его восприимчивость к пульсациям внешнего потока, возбуждение и эволюцию 
неустойчивых возмущений и в конечном счете влиять на положение перехода ламинарного течения в турбулентное.

Реальные ГЛА имеют затупленные носовые части и передние кромки. При обтекании затупленных тел на сверхзвуковых скоростях образуется искривленная головная ударная волна и, как следствие, зона энтропийного слоя. Известно, что небольшие радиусы затупления приводят к стабилизации пограничного слоя [2] и сдвигают ЛТП вниз по потоку, при этом большие радиусы затупления имеют обратный эффект (так называемый реверс перехода). Влияние локального нагрева на средние характеристики пограничного слоя и ЛТП в присутствии энтропийного слоя может оказаться критичным для проектирования ГЛА.

Для компоновок, имеющих аэродинамически гладкие поверхности, переход связан с возбуждением и усилением в пограничном слое неустойчивых возмущений, наиболее опасные из которых первая и вторая моды. Линейное развитие этих мод достаточно хорошо изучено. Известно, что нагрев, равномерно распределенный по всей поверхности обтекаемой потоком газа модели, приводит при гиперзвуковых скоростях к дестабилизации первой моды возмущений и стабилизации второй. Охлаждение производит противоположный эффект [3].

При локальном воздействии температуры поверхности на переход в поведении возмущений возникают особенности [4]. Так, при экспериментальном исследовании локального воздействия нагрева/охлаждения поверхности вдали от носовой части на переход гиперзвукового пограничного слоя на остром конусе было обнаружено, что локальное охлаждение стабилизирует пограничный слой и сдвигает ЛТП вниз по потоку. Локальный нагрев дестабилизирует пограничный слой и сдвигает ЛТП вверх по потоку [5]. Таким образом, получен эффект, прямо противоположный равномерному нагреву или охлаждению всей поверхности. С другой стороны, локально нагретый газ, который сносится вниз по потоку, приводит к эффективному снижению температурного фактора (отношения температуры поверхности к температуре газа), т. е. могут возникать локальные зоны эффективного охлаждения.

Влияние одновременно двух факторов - притупления носика и его нагрева/охлаждения - на характеристики ЛТП до сих пор не изучалось. В настоящей работе представлены первые результаты экспериментального исследования влияния локального нагрева/охлаждения затупленной носовой части конуса на развитие возмущений в гиперзвуковом пограничном слое.

Письма в ЖТФ, 2017, том 43, вып. 20 
Экспериментальные исследования проводились в гиперзвуковой аэродинамической установке „Транзит-М“ ИТПМ СО РАН [6] при числе Maха $M=6$, температуре торможения $T_{0}=360-418 \mathrm{~K}$ и давлении торможения $P_{0}=3.7-46 \mathrm{~atm}$. Экспериментальное исследование развития возмущений было проведено в пограничном слое конусов с углом полураствора $7^{\circ}$ и длиной $459 \mathrm{~mm}$ (для острого носика) с различными радиусами затупления носовой части: $R=0.1 \mathrm{~mm}$ (острый, длина $65 \mathrm{~mm}$ ), $0.75 \mathrm{~mm}$ (длина $60 \mathrm{~mm}$ ) и $1.5 \mathrm{~mm}$ (длина $54 \mathrm{~mm}$ ). Сменные металлические носики с различными радиусами затупления были изготовлены с возможностью нагрева и охлаждения их внутренней части. Нагрев осуществлялся с помощью омического нагревателя, охлаждение подводом жидкого азота во внутреннюю полость носовой части модели. Зона нагрева и охлаждения была ограничена длиной носика, за счет хороших теплоизоляционных свойств материала модели и небольшого времени нагрева растекание тепла (градиент температуры) при нагреве заняло около $20 \mathrm{~mm}$, при охлаждении - 50-60 mm. Основное тело модели было выполнено из высокотемпературного пластика РЕЕК материала, сочетающего достаточно высокую механическую прочность с высокой температурой ее потери. Низкий уровень теплопроводности позволил обеспечить высокий пространственный градиент температуры на поверхности модели. РЕЕК имеет степень черноты 0.95 , что позволило выполнить измерения температуры поверхности с помощью тепловизора.

Эксперименты проводились при различных температурах носика: $T_{w} \approx 440,300,90 \mathrm{~K} ; T_{w} / T_{r} \approx 1.33,0.9,0.3$ соответственно $\left(T_{r}\right.$ - температура восстановления), при этом температура поверхности остальной модели была равна $\sim 300 \mathrm{~K}, T_{w} / T_{r} \approx 0.9$. Значение $T_{w} / T_{r} \approx 0.9$, близкое к адиабатическому нагреву поверхности конуса, принималось за базовое. Минимальная температура в случае охлаждения была близка к температуре кипения жидкого азота, максимальная температура нагрева была ограничена температурой потери механической прочности материала РЕЕК. Время, требуемое для достижения максимальной/минимальной температуры, составляло примерно 80/210 s.

Для измерения пульсаций давления на модели заподлицо с поверхностью были установлены высокочастотные пьезодатчики давления PCB 132A31. Датчики РCB1-PCB5 установлены по образующей (на расстоянии $\sim 164,219,284,349,414 \mathrm{~mm}$ от начала острого носика), датчик PCB6 повернут на $180^{\circ}$ относительно датчика PCB4 для контроля угла атаки. Сбор данных осуществлялся двумя четырехканальными

Письма в ЖТФ, 2017, том 43, вып. 20 
модулями аналого-цифрового преобразователя L-card E20-10 с частотой до $1.67 \mathrm{MHz}$. Распределение температуры поверхности контролировалось тепловизором Flir.

Обработка данных эксперимента осуществлялась в небольших временнб́х интервалах, в которых параметры потока могли считаться квазистационарными. Спектральные распределения вычислялись осреднением по $l$ блокам по $2^{n}$ точек в каждом блоке во временно́м интервале $170-180 \mathrm{~ms}$ от начала пуска. Число блоков равнялось 65, при количестве точек в блоке 256.

Эксперименты с острым носиком проведены для единичных чисел Рейнольдса $\operatorname{Re}_{1} \sim 4.7 \cdot 10^{6}$ и $\sim 13 \cdot 10^{6} 1 / \mathrm{m}$. Для обоих чисел Рейнольдса результаты качественно совпали.

Проведем сравнительный анализ спектров пульсаций давления в пограничном слое для $\operatorname{Re}_{1} \approx 13 \cdot 10^{6} 1 / \mathrm{m}$. Измерения показали, что ЛТП в пограничном слое произошел в области датчика РСВ4. Проследим эволюцию возмущений в предпереходной области (выше по течению от этого датчика).

Для датчика PCB1 на спектрах пульсаций давления явно наблюдается пик второй моды как для базового случая, так и для нагрева. Идентификация второй моды во всех экспериментах, представленных в настоящей работе, выполнялась по наличию характерных черт, которые ее описывают. Вторая мода относится к высокочастотным коротковолновым возмущениям. Ее характерной чертой является выполнение условия $\lambda \approx 2 \delta$, где $\lambda-$ длина волны возмущения, $\delta-$ толщина пограничного слоя. Выполнение этого соотношения и соответствие безразмерного частотного параметра диапазону безразмерных частот второй моды проверялись для всех спектров, представленных в работе. При нагреве в спектрах пульсаций давления, измеренных датчиком PCB1, наблюдается увеличение амплитуды пика второй моды. Пульсации давления в диапазоне частот $f \approx 50-180 \mathrm{kHz}$ увеличиваются. В случае охлаждения носика модели возмущений второй моды не наблюдается.

Наиболее отчетливо результаты этих изменений видны в спектрах датчика РСВ2, приведенных на рисунке, $a$. Можно видеть, что спектры пульсаций давления в диапазоне частот $f \approx 50-200 \mathrm{kHz}$ для охлаждения явно ниже спектров базового случая и нагрева. Охлаждение стабилизирует развитие возмущений. Изменение частоты пика происходит вследствие изменения толщины пограничного слоя вниз по потоку.

Письма в ЖТФ, 2017, том 43, вып. 20 

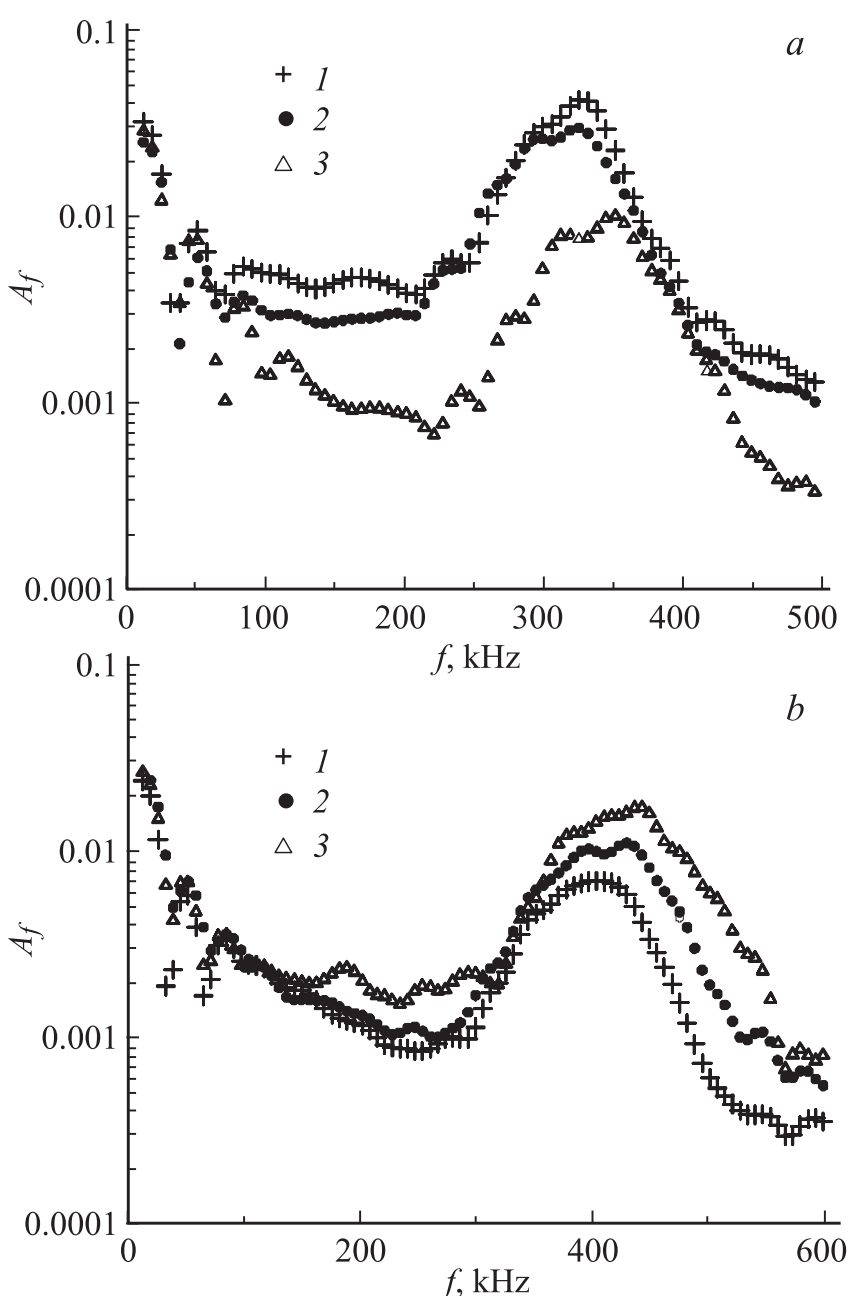

Спектры пульсаций давления при разных $T_{w} / T_{r}$, полученные для: $\left.a\right) R=0.1 \mathrm{~mm}$, $x=218.6 \mathrm{~mm} \quad$ (PCB2). $1-\operatorname{Re}_{1} \approx 13.1 \cdot 10^{6} 1 / \mathrm{m}, \quad T_{w} / T_{r} \approx 1.33 ; 2-$ $\operatorname{Re}_{1} \approx 13 \cdot 10^{6} 1 / \mathrm{m}, \quad T_{w} / T_{r} \approx 0.89 ; 3-\mathrm{Re}_{1} \approx 12.9 \cdot 10^{6} 1 / \mathrm{m}, \quad T_{w} / T_{r} \approx 0.29$. b) $R=0.75 \mathrm{~mm}, x=218.6 \mathrm{~mm}$ (PCB2). $1-\mathrm{Re}_{1} \approx 19.5 \cdot 10^{6} 1 / \mathrm{m}, T_{w} / T_{r} \approx 1.25$; $2-\mathrm{Re}_{1} \approx 19.8 \cdot 10^{6} 1 / \mathrm{m}, T_{w} / T_{r} \approx 0.87 ; 3-\mathrm{Re}_{1} \approx 19.9 \cdot 10^{6} 1 / \mathrm{m}, T_{w} / T_{r} \approx 0.3$. c) $R=1.5 \mathrm{~mm}, x=283.6 \mathrm{~mm}$ (PCB3). $1-\mathrm{Re}_{1} \approx 32.8 \cdot 10^{6} 1 / \mathrm{m}, T_{w} / T_{r} \approx 1.39$; $2-\mathrm{Re}_{1} \approx 32.5 \cdot 10^{6} 1 / \mathrm{m}, T_{w} / T_{r} \approx 0.92 ; 3-\mathrm{Re}_{1} \approx 31.9 \cdot 10^{6} 1 / \mathrm{m}, T_{w} / T_{r} \approx 0.29$. 


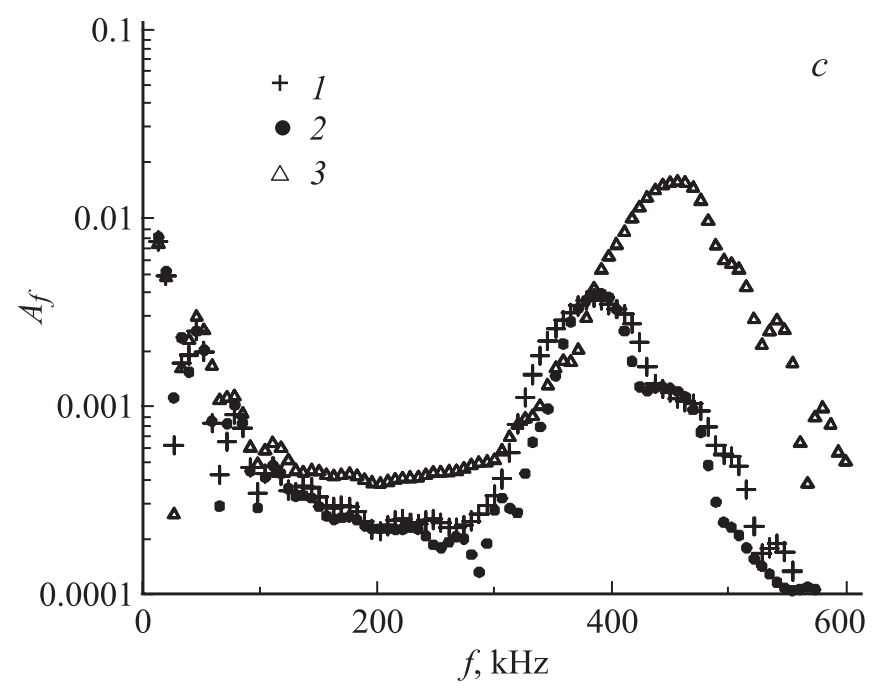

Рисунок (продолжсение).

Как в базовом случае, так и в случае нагрева пики второй моды имеют бо́льшую амплитуду, а также происходит усиление пульсаций для частот $f \approx 50-150 \mathrm{kHz}$.

Для координаты, где расположен датчик РСВ3, спектры пульсаций для всех температур очень близки, а датчик РCB5 фиксирует, что все спектры практически совпадают, пиков в спектрах не наблюдается, что характерно для турбулентного течения, т.е. ЛТП в пограничном слое произошел в области датчика РСB4.

На основании наблюдения за спектрами можно заключить, что нагрев носовой части острого конуса слабо дестабилизирует вторую моду возмущения, а охлаждение - стабилизирует. Такой же эффект наблюдается для небольших чисел Рейнольдса $\left(\operatorname{Re}_{1} \approx 4.7 \cdot 10^{6} 1 / \mathrm{m}\right)$. Здесь следует отметить, что увеличение единичного числа Рейнольдса приводит к тому, что безразмерная область влияния нагрева/охлаждения уменьшается, но это не оказывает влияния на качественное поведение спектров. Получен эффект, прямо противоположный равномерному влиянию температуры поверхности на развитие возмущений в гиперзвуковом пограничном слое, при котором нагрев стабилизирует,

2 Письма в ЖТФ, 2017, том 43, вып. 20 
а охлаждение дестабилизирует вторую моду. Этот вывод согласуется с результатом работы [4].

На рисунке, $b$ представлены спектры пульсаций давления для датчика РCВ2, полученные на конусе с затуплением $R=0.75 \mathrm{~mm}$. Видно, что для всех трех температур поверхности в частотном диапазоне $f \approx 300-550 \mathrm{kHz}$ наблюдается вторая мода. Максимальная величина пика второй моды соответствует охлаждению поверхности, а минимальная - нагреву. Таким образом, охлаждение дестабилизирует течение, нагрев - стабилизирует. Данное поведение развития возмущений противоположно поведению возмущений в модели с остроконечным носиком.

Для датчика РСВ3 в базовом случае и при охлаждении происходит рост амплитуд всех частот до частоты второй моды, т.е. турбулизация течения, а при нагреве - уменьшение пульсаций в частотном диапазоне $f \approx 50-200 \mathrm{kHz}$. Ниже по потоку в спектрах, регистрируемых датчиком PCB4, пульсации для базового случая и охлаждения имеют примерно одинаковый уровень. Спектры пологие, без пиков, что характерно для турбулентного потока.

Схожее поведение спектров наблюдается при увеличении радиуса затупления до $1.5 \mathrm{~mm}$. Спектры пульсаций давления, регистрируемые датчиком РСВ2, совпадают для всех трех температур носика конуса. Поведение спектров, полученных при измерениях датчиком РСВ3 (см. рисунок, $c$ ), качественно совпадает с поведением спектров, представленных на рисунке, $b$ для $R=0.75 \mathrm{~mm}$. Для базового случая в спектре пульсаций давления наблюдается явно выраженный пик второй моды для $f \approx 390 \mathrm{kHz}$. Видно, что нагрев слабо влияет на спектр, a охлаждение дестабилизирует течение: амплитуда пульсаций второй моды примерно в 4 раза больше. Кроме того, для случая охлаждения носика видно увеличение амплитуд в спектрах пульсаций давления на частотах $f \approx 50-300 \mathrm{kHz}$. Эффект охлаждения для $R=1.5 \mathrm{~mm}$ проявляется сильнее, чем в случае $R=0.75 \mathrm{~mm}$.

В целом можно заключить, что для конуса с притуплением нагрев носика слабо влияет на пульсации в гиперзвуковом пограничном слое, а охлаждение приводит к заметной дестабилизации возмущений. Этот эффект противоположен влиянию нагрева/охлаждения на возмущения в пограничном слое острого конуса и аналогичен влиянию равномерного нагрева/охлаждения. Обнаруженные эффекты более явно проявляются при большем радиусе притупления.

Письма в ЖТФ, 2017, том 43, вып. 20 
Для более полного ответа на вопрос, как влияет изменение температурного фактора носика модели на ЛТП, необходимо более точно определить положение ЛТП для всех рассматриваемых случаев.

Итак, сравнительный анализ спектров пульсаций давления в пограничном слое показал, что в пограничном слое острого конуса нагрев/охлаждение приводит к дестабилизации/стабилизации возмущений второй моды. Получен эффект, прямо противоположный влиянию равномерной температуры поверхности на развитие возмущений в гиперзвуковом пограничном слое, при котором нагрев стабилизирует, а охлаждение дестабилизирует вторую моду.

Проведены исследования в пограничном слое двух затупленных конусов с радиусами затупления 0.75 и $1.5 \mathrm{~mm}$. Для затупленных конусов наблюдается эффект, противоположный результатам, полученным для острого конуса: нагрев слабо стабилизирует возмущения в гиперзвуковом пограничном слое, охлаждение приводит к дестабилизации возмущений, что совпадает с влиянием равномерного охлаждения. Эффект усиливается при увеличении радиуса затупления носовой части конуса. Несмотря на то что при разных единичных числах Рейнольдса безразмерная область влияния нагрева/охлаждения различна, качественное поведение спектров для разных $\mathrm{Re}_{1}$ совпало для всех радиусов притупления.

Работа выполнена при поддержке РФФИ (грант № 15-01-04788).

\section{Список литературы}

[1] Бойко А.В., Кириловский С.В., Маслов А.А., Поплавская Т.В. // ПМТФ. 2015. T. 56. № 5. C. 30-49.

[2] Maslov A.A., Shiplyuk A.N., Buntin D.A., Sidorenko A.A. // J. Spacecraft Rockets. 2006. V. 43. P. 71-76.

[3] Lysenko V.I., Maslov A.A. // J. Fluid Mechanics. 1984. V. 147. P. 39-52.

[4] Soudakov V.G., Egorov I.V., Fedorov A.V. // Proc. of the 6th European Symp. on aerothermodynamics for space vehicles. Versailles, France, 2008. ESA SP-659.

[5] Fedorov A., Soudakov V., Egorov I., Sidorenko A., Gromyko Y., Bountin D., Polivanov P., Maslov A. // AIAA Journal. 2015. V. 53. Pt 9. P. 2512-2524.

[6] Fomin V.M., Kharitonov A.M., Maslov A.A., Shiplyuk A.N., Shumskii V.V., Yaroslavtsev M.I., Zvegintsev V.I. // Experiment. methods of shock wave research. Berlin-Heidelberg-N. Y.: Springer-Verlag, 2016. V. 9. P. 315-346.

2* Письма в ЖТФ, 2017, том 43, вып. 20 